PROCEEDINGS OF THE

AMERICAN MATHEMATICAL SOCIETY

Volume 129, Number 7 , Pages 1949-1953

S 0002-9939(01)05918-4

Article electronically published on February 22, 2001

\title{
CONJUGACIES BETWEEN RATIONAL MAPS AND EXTREMAL QUASICONFORMAL MAPS
}

\author{
GUIZHEN CUI
}

(Communicated by Michael Handel)

\begin{abstract}
We show that two rational maps which are $K$-quasiconformally combinatorially equivalent are $K$-quasiconformally conjugate. We also study the relationship between the boundary dilatation of a combinatorial equivalence and the dilatation of a conjugacy.
\end{abstract}

The Teichmüller theory is a powerful tool in the study of complex analytic dynamics. In addition to the work of D. Sullivan $[\mathrm{Su}]$ and W. Thurston (refer [DH2]), where the classical theory of the Teichmüller spaces plays a crucial role, a beautiful theorem about extremal quasiconformal maps between open Riemann surfaces, due to R. Strebel $([\underline{\mathrm{S}}])$, was employed in the work of C. McMullen [Mc2]. In this paper, we give new applications of this theorem and a theorem of $\mathrm{H}$. Ohtake $(\mathrm{Oh}])$ about lifts of extremal quasiconformal maps.

Suppose that $f, g$ are rational maps of degree bigger than one which are quasiconformally combinatorially equivalent, i.e. there exist quasiconformal maps $\phi$ and $\phi_{1}$ from $\hat{\mathbb{C}}$ to itself such that $\phi$ is isotopic to $\phi_{1}$ rel $P(f)$ and $\phi f=g \phi_{1}$. Here

$$
P(f)=\overline{\bigcup_{n>0} f^{n}\left(C_{f}\right)}
$$

is the post-critical set of $f$, where $C_{f}$ is the set of the critical points of $f$. It is known that there exists a quasiconformal conjugacy $\psi$ from $f$ to $g$, which is isotopic to $\phi$ rel $P(f)$ (Mc3]). In the proof, $\psi$ was constructed by the DouadyEarle extension and hence its maximal dilatation might be bigger than the maximal dilatation of $\phi$. Applying extremal quasiconformal maps, we improve the theorem to show that $\psi$ can be chosen such that its maximal dilatation is less than or equal to the maximal dilatation of $\phi$ (Theorem 1) and we give an application (Theorem 2) relating extremal and boundary dilatation.

At first, let us recall Strebel's Theorem. Let $\phi: R \rightarrow R^{\prime}$ be a quasiconformal map between open Riemann surfaces. The Beltrami differential of $\phi$ is

$$
\mu_{\phi}(z) \frac{d \bar{z}}{d z}=\frac{\partial_{\bar{z}} \phi(z)}{\partial_{z} \phi(z)}
$$

Received by the editors August 21, 1999.

2000 Mathematics Subject Classification. Primary 37F30, 30C75.

Key words and phrases. Rational map, extremal quasiconformal map.

This work is supported by the National Natural Sience Foundation of China (No. 19871084). 
and the maximal dilatation of $\phi$ is

$$
K(\phi)=\frac{1+\left\|\mu_{\phi}\right\|_{\infty}}{1-\left\|\mu_{\phi}\right\|_{\infty}} .
$$

The boundary dilatation $K_{1}[\phi]$ of $\phi$ is the infimum of the maximal dilatation of $\left.\psi\right|_{W}$ over all quasiconformal maps $\psi$ isotopic to $\phi$ rel the boundary and all neighborhoods $W$ of $\partial R$.

The map $\phi$ is called extremal if $K(\phi) \leq K(\psi)$ for all quasiconformal maps $\psi$ isotopic to $\phi$ rel the boundary.

Let $q(z) d z^{2}$ be an integrable holomorphic quadratic differential on $R$, i.e.

$$
\int_{R}|q(z)| d x d y<\infty
$$

A quasiconformal map $\phi$ is called a Teichmüller map associated with $q$ if

$$
\mu_{\phi}(z)=k \frac{\overline{q(z)}}{|q(z)|}
$$

for some constant $0<k<1$. A Teichmüller map is the unique extremal map.

Strebel's Theorem. Let $\phi$ be an extremal quasiconformal map with $K(\phi)>1$. If $K_{1}[\phi]<K(\phi)$, then $\phi$ is a Teichmüller map associated with an integrable holomorphic quadratic differential on $R$.

Theorem 1. Let $f, g$ be rational maps of degree bigger than one. Suppose that $\phi, \phi_{1}$ are $K$-quasiconformal maps from $\hat{\mathbb{C}}$ to itself such that $\phi_{1}$ is isotopic to $\phi$ rel $P(f)$ and $\phi f=g \phi_{1}$ on $\hat{\mathbb{C}}$. Then there exists a $K$-quasiconformal conjugacy $\psi$ from $f$ to $g$ which is isotopic to $\phi$ rel $P(f)$.

Proof. Clearly, $\phi(P(f))=P(g)$ and the theorem holds when $P(f)$ consists of two points. So assume that $P(f)$ contains at least three points. Denote

$$
Q(f)=\overline{\bigcup_{n>0} f^{-n}(P(f))} .
$$

The Julia set $J(f)$ of $f$ is contained in $Q(f)$.

There exists a lift $\phi_{2}$ of $\phi_{1}$, such that $\phi_{2}$ is isotopic to $\phi_{1}$ rel $f^{-1}(P(f))$ and $\phi_{1} f=g \phi_{2}$. Continuing this process, we get a sequence of $K$-quasiconformal maps $\left\{\phi_{n}\right\}$, such that $\phi_{n+1}$ is isotopic to $\phi_{n}$ rel $f^{-n}(P(f))$ and $\phi_{n} f=g \phi_{n+1}$. There exist subsequences $\left\{\phi_{n_{i}}\right\},\left\{\phi_{n_{i}+1}\right\}$, which uniformly converge to $K$-quasiconformal maps $\Phi$ and $\Phi_{1}$, respectively. Clearly, $\Phi(Q(f))=Q(g), \Phi_{1}$ is isotopic to $\Phi$ rel $Q(f)$ and $\Phi f=g \Phi_{1}$.

Suppose that $D_{1}$ is a periodic Fatou domain of $f$ with period $p \geq 1$. Then $D_{2}=\Phi\left(D_{1}\right)=\Phi_{1}\left(D_{1}\right)$ is a periodic Fatou domain of $g$ with the same type and period as $D_{1}$. We claim that there exists a $K$-quasiconformal map $\psi$ from $D_{1}$ to $D_{2}$, such that $\psi$ is isotopic to $\left.\Phi\right|_{D_{1}}$ rel $Q(f)$ and $\psi f^{p}=g^{p} \psi$. (Note that a quasiconformal isotopy rel ideal boundary is equivalent to a quasiconformal isotopy rel real boundary, by [EM].)

Case 1. $D_{1}$ is a Siegel disk or a Herman ring. $D_{1}-Q(f)$ is a disk $U_{1}$ or a disjoint union of annuli $U_{1}, \cdots, U_{m}$ and $f^{p}\left(U_{i}\right)=U_{i}$. The continuous extensions of $f^{p}, g^{p}$ to the ideal boundary of $U_{i}, \Phi\left(U_{i}\right)$, denoted by $\bar{f}^{p}$ and $\bar{g}^{p}$, are irrational rotations. Let $\bar{\Phi}$ and $\bar{\Phi}_{1}$ denote the continuous extensions of $\Phi$ and $\Phi_{1}$ to the ideal boundary, respectively. Then $\bar{\Phi}=\bar{\Phi}_{1}$ and $\bar{\Phi} \bar{f}^{p}=\bar{g}^{p} \bar{\Phi}_{1}$. Now $\bar{f}^{p}$ and $\bar{g}^{p}$ are irrational rotations 
with the same rotation number since they are topologically conjugate. Thus $\bar{\Phi}$ is a rotation since the orbit of every point is dense in each component of the ideal boundary.

By Strebel's Theorem, there exists a unique extremal quasiconformal map $\psi_{i}$ in the isotopy class of $\left.\Phi\right|_{U_{i}}$. Thus $\psi_{i} f^{p}=g^{p} \psi_{i}$. Let $\psi=\psi_{i}$ on each $U_{i}$ and $\psi=\Phi$ on $Q(f)$. Then $\psi$ is a $K$-quasiconformal map from $D_{1}$ to $D_{2}, \psi$ is isotopic to $\left.\Phi\right|_{D_{1}}$ rel $Q(f)$ and $\psi f^{p}=g^{p} \psi$.

Case 2. $D_{1}$ is superattracting. $D_{1}-Q(f)$ is a punctured disk $U_{1}$ or a disjoint union of annuli $U_{i}, i \in \mathbb{Z}$. Identify each component of the ideal boundary of $U_{i}, \Phi\left(U_{i}\right)$, with the unit circle $S^{1}$. The continuous extensions of $f^{p}$ and $g^{p}, \bar{f}^{p}$ and $\bar{g}^{p}$, are $z \rightarrow z^{d}$ on $S^{1}$ for some $d>1$. Let $\bar{\Phi}$ and $\bar{\Phi}_{1}$ denote the continuous extensions of $\Phi$ and $\Phi_{1}$ to the ideal boundary, respectively. Then $\bar{\Phi}=\bar{\Phi}_{1}$ and $\bar{\Phi} \bar{f}^{p}=\bar{g}^{p} \bar{\Phi}_{1}$. Thus $\bar{\Phi}$ is a rotation.

An argument similar to the one above shows that there is a $K$-quasiconformal map $\psi$ from $D_{1}$ to $D_{2}$ such that $\psi$ is isotopic to $\left.\Phi\right|_{D_{1}}$ rel $Q(f)$ and $\psi f^{p}=g^{p} \psi$.

Case 3. $D_{1}$ is attracting. There exist a domain $V_{i} \subset D_{i}, i=1,2$, which contains the periodic point, and a conformal map $h_{i}$ from $V_{i}$ into $\mathbb{C}$, such that $h_{i}$ maps the periodic point to the origin,

$$
\begin{gathered}
h_{1} f^{p} h_{1}^{-1}(z)=\lambda_{1}(z)=\lambda_{1} z \quad \text { and } \\
h_{2} g^{p} h_{2}^{-1}(z)=\lambda_{2}(z)=\lambda_{2} z
\end{gathered}
$$

whenever they are defined. Denote

$$
\begin{gathered}
E_{1}=h_{1}\left(Q(f) \cap V_{1}\right) \quad E_{2}=h_{2}\left(Q(g) \cap V_{2}\right) \\
\text { and } K_{i}=\bigcup_{n>0} \lambda_{i}^{-n}\left(E_{i}\right) .
\end{gathered}
$$

Then $\lambda_{i}\left(K_{i}\right)=K_{i}$ and $R_{i}=\left(\mathbb{C}-K_{i}\right) /\left\langle\lambda_{i}\right\rangle$ is a punctured torus, where $\left\langle\lambda_{i}\right\rangle$ means the Abelian group generated by $z \rightarrow \lambda_{i} z$.

Now $h_{2} \Phi h_{1}^{-1}\left(\lambda_{1} z\right)=\lambda_{2} h_{2} \Phi_{1} h_{1}^{-1}(z)$ is $K$-quasiconformal on a neighborhood of the origin. There exists a subsequence of $\left\{\lambda_{2}^{-n} h_{2} \Phi h_{1}^{-1} \lambda_{1}^{n}\right\}_{n=1}^{\infty}$ which uniformly converges to a $K$-quasiconformal map $\Psi$ of $\mathbb{C}$. Let $\Psi_{1}(z)=\lambda_{2}^{-1} \Psi\left(\lambda_{1} z\right)$; then $\Psi_{1}$ is isotopic to $\Psi \operatorname{rel} K_{1}$.

$\Psi$ defines an isotopy class of homeomorphisms from $R_{1}$ to $R_{2}$. Let $\tilde{\Psi}_{0}$ be the unique extremal quasiconformal map in this isotopy class. Then $\tilde{\Psi}_{0}$ is $K$ quasiconformal since $\mathbb{C}-K_{i} \rightarrow R_{i}$ is a normal covering with Abelian transformation group (refer to $[\mathrm{Oh}]$ ). Lifting $\tilde{\Psi}_{0}$, we get a $K$-quasiconformal map $\Psi_{0}$ of $\mathbb{C}$ such that $\Psi_{0}$ is isotopic to $\Psi$ rel $K_{1}$ and $\Psi_{0}\left(\lambda_{1} z\right)=\lambda_{2} \Psi_{0}(z)$.

Let $\psi_{0}=h_{2}^{-1} \Psi_{0} h_{1}$ and pullback $\psi_{0}$ along $f^{n p}$ and $g^{n p}$ to the Fatou domain $D_{1}$. We get a $K$-quasiconformal map $\psi$ from $D_{1}$ to $D_{2}$ such that $\psi$ is isotopic to $\left.\Phi\right|_{D_{1}}$ rel $Q(f)$ and $\psi f^{p}=g^{p} \psi$.

Case 4. $D_{1}$ is parabolic associated to a parabolic periodic point $z_{1}$. The quasiconformality of $\Phi$ implies that there exists an attracting petal $V_{1} \subset D_{1}$ such that $\Phi\left(V_{1}\right)$ is contained in an attracting petal $D_{2}$. By an attracting petal $V_{1} \subset D_{1}$, we mean that $f^{p}(\bar{V}) \subset V \cup\left\{z_{1}\right\}$ and for any $z \in D_{1}, f^{n}(z) \in V$ for some $n>0$. A similar argument as in Case 3 shows the existence of a $K$-quasiconformal conjugacy $\psi$ from $\left(f^{p}, D_{1}\right)$ to $\left(g^{p}, D_{2}\right)$ in the isotopy class of $\left.\Phi\right|_{D_{1}}$ rel $Q(f)$. 
Now pullback $\psi$ along $f^{n}$ and $g^{n}$. We get a $K$-quasiconformal conjugacy $\psi$ from $(f, F(f))$ to $(g, F(g))$ in the isotopy class of $\left.\Phi\right|_{F(f)}$ rel $Q(f)$. Let $\psi=\Phi$ on $J(f)$. Then $\psi$ is a $K$-quasiconformal map of $\hat{\mathbb{C}}$ by Rickman's Theorem (refer to [Ri] or DH1], p. 303, Lemma 2), $\psi$ is isotopic to $\phi$ rel $P(f)$ and $\psi f=g \psi$ on $\hat{\mathbb{C}}$.

We now give an application. Let $\phi:(\hat{\mathbb{C}}, P(f)) \rightarrow(\hat{\mathbb{C}}, P(g))$ be a quasiconformal combinatorial equivalence between rational maps $f, g$. Denote by $K_{0}[\phi]$ the infimum of the maximal dilatations over all quasiconformal conjugacies between $f$ and $g$ in the isotopy class of $\phi$. Denote by $K_{1}[\phi]$ the infimum of the maximal dilatations of $\left.\psi\right|_{W}$ over the quasiconformal maps $\psi$ in the isotopy class of $\phi$ and all open sets $W \supset P(f)$. Recall that $K_{1}[\phi]$ is the boundary dilatation of $\phi$ on $\hat{\mathbb{C}}-P(f)$ if $P(f) \neq \hat{\mathbb{C}}$. Obviously, $K_{1}[\phi] \leq K_{0}[\phi]$. An interesting problem is to characterize when equality holds.

Examples. 1. $K_{1}[\phi]=K_{0}[\phi]=0$ whenever $f$ and $g$ are postcritically finite and not covered by integral torus endomorphisms, since Thurston rigidity for such maps implies that they are in fact globally conformally conjugate. So equality holds in this case.

2. Integral torus endomorphisms have a one-parameter family of deformations, so in this case $K_{1}[\phi]=0$ but $K_{0}[\phi]>0$.

3. If $f$ has a Herman ring or a Siegel disc containing a point of $P(f)$ with infinite forward orbit, then one can deform $f$ by fattening an annulus compactly contained in the interior of the Siegel disc or Herman ring. Thus again one can produce $g$ and $\phi$ for which $K_{1}[\phi]=0$ while $K_{0}[\phi]>0$.

The last two examples for which equality need not hold have the property that the rational maps contain Thurston obstruction; see [Mc1]. The following theorem shows that in the absence of Thurston obstruction we have equality.

Theorem 2. Suppose that $f$ is a rational map which has no Thurston obstruction. If $f$ and $g$ are combinatorially equivalent via a quasiconformal map $\phi$, then $K_{0}[\phi]=$ $K_{1}[\phi]$.

Proof. Suppose that $K_{0}[\phi]=K_{1}[\phi]+2 \epsilon$ for some $\epsilon>0$ otherwise. Let $\phi_{0}$ be a quasiconformal map in the isotopy class of $\phi$ such that $\phi_{0}$ is extremal on each component of $\hat{\mathbb{C}}-P(f)$. Then $K\left(\phi_{0}\right)=K_{0}[\phi]$ by Theorem 1 . Let $K_{U}$ be the maximal dilatation of the restriction of $\phi_{0}$ to the component $U$ of $\hat{\mathbb{C}}-P(f)$. By Strebel's Theorem, either $K_{U} \leq K_{1}[\phi]+\epsilon$, or $\left.\phi_{0}\right|_{U}$ is a Teichmüller map and hence is the unique extremal map. By the definition of $K_{1}[\phi]$, there are at most finitely many components such that $K_{U}>K_{1}[\phi]+\epsilon$. Let $V$ be a component such that $K_{V} \geq K_{U}$ for all the components $U$. Then $K_{V}=K\left(\phi_{0}\right)=K_{0}[\phi]$.

There exists a quasiconformal map $\psi_{0}$ in the isotopy class of $\phi$ such that $K\left(\left.\psi_{0}\right|_{W}\right)$ $<K_{1}[\phi]+\epsilon=K_{0}[\phi]-\epsilon$, where $W$ is a neighborhood of $P(f)$. Let $\psi_{k}$ be the isotopic lift of $\psi_{k-1}$ with $\psi_{k-1} f=g \psi_{k}$. Then $K\left(\left.\psi_{k}\right|_{f^{-k}(W)}\right)=K\left(\left.\psi_{0}\right|_{W}\right)$. Let $\Psi$ be a limit of the sequence $\left\{\psi_{k}\right\}$. Then $K\left(\left.\Psi\right|_{D}\right) \leq K\left(\left.\psi_{0}\right|_{W}\right) \leq K_{0}[\phi]-\epsilon$ for each attracting, superattracting or parabolic domain $D$ of $f$ since any compact set in $D$ is contained in $f^{-k}(W)$ for sufficiently large integer $k$. Moreover, $\Psi$ is isotopic to $\phi$ rel $Q(f)$. Thus $V$ is not contained in attracting, superattracting and parabolic domains.

Since there is no Thurston obstruction for $f, f$ has no Herman ring and a Siegel disk of $f$ (if it exists) contains no $P(f)$ point except the center. By the argument 
in Case 1 of the proof of Theorem 1, $\left.\phi_{0}\right|_{U}$ is conformal when $U$ is contained in a Siegel disc. So $V \cap J(f) \neq \emptyset$.

Let $\phi_{k}(k \geq 1)$ be the isotopic lift of $\phi_{k-1}$ with $\phi_{k-1} f=g \phi_{k}$. Then $K\left(\phi_{k}\right)=$ $K\left(\phi_{0}\right)$ and hence $\left.\phi_{k}\right|_{V}=\left.\phi_{0}\right|_{V}$ since $\left.\phi_{0}\right|_{V}$ is a Teichmüller map. For each component $U$, since $V \cap J(f) \neq \emptyset$, there exists a domain $W \subset V$ such that $f^{k}(W) \subset U$ for some $k>0$. Thus $K_{U} \geq K\left(\left.\phi_{k}\right|_{W}\right)=K_{V}$ since $\left.\phi_{k}\right|_{V}$ is a Teichmüller map. Thus $\left.\phi_{0}\right|_{U}$ is also a Teichmüller map with constant maximal dilatation and $\phi_{k}=\phi_{0}$ for all integers $k>0$, i.e. $\phi_{0}$ is actually a conjugacy.

If the Fatou set $F(f)$ of $f$ is nonempty, then the restriction of $\phi_{0}$ to each component of $F(f)-Q(f)$ is a Teichmüller map and hence is the unique extremal map. We know $f$ has no Herman ring. If $f$ has a Siegel disk $D$, then $\left.\phi_{0}\right|_{D}$ is conformal. This is a contradiction. If $f$ has an attracting, superattracting or parabolic domain $D$, then $K\left(\left.\Psi\right|_{D}\right) \leq K\left(\phi_{0}\right)-\epsilon$. This is also a contradiction. Hence $J(f)=\hat{\mathbb{C}}$.

By Lemma 3.9 in [Mc1], $f$ is critically finite, the signature of the orbifold of $f$ is $(2,2,2,2)$ and $f$ is covered by an integral torus endomorphism, i.e. there is a Thurston obstruction for $f$. It is a contradiction.

\section{ACKNOWLEDGEMENT}

The author thanks the referee for kind suggestions and corrections.

\section{REFERENCES}

[EM] C. Earle and C. McMullen, Quasiconformal isotopies, In Holomorphic Functions and Moduli I, Springer-Verlag, MSRI publications 10 (1988), 143-154. MR 89h:30028

[DH1] A. Douady and J. Hubbard, On the dynamics of polynomial-like mappings, Ann. Sci. Éc. Norm. Sup. 18 (1985), 287-344. MR 87f:58083

[DH2] A. Douady and J. Hubbard, A proof of Thurston's topological characterization of rational maps, Acta Math. 171 (1993), 263-297. MR 94j:58143

[Mc1] C. McMullen, Complex dynamics and Renormalization, Annals of Math Studies 135, Princeton University Press, 1994. MR 96b:58097

[Mc2] - Automorphisms of rational maps, In Holomorphic Functions and Moduli I, Springer-Verlag, MSRI publications 10 (1988), 31-60. MR 89m:58187

[Mc3] _ Self-similarity of Siegel disks and Hausdorff dimension of Julia sets, Acta Math. 180 (1998), 247-292. MR 99f:58172

[Oh] H. Ohtake, Lifts of extremal quasiconformal mappings of arbitrary Riemann surfaces, J. Math. Kyoto Univ. 22 (1982), 191-200. MR 84e:30071

[Ri] S. Rickman, Removability theorems for quasiconformal mappings, Ann. Ac. Scient. Fenn. 449 (1969), 1-8. MR 40:7443

[S] K. Strebel, On quasiconformal mappings of open Riemann surfaces, Comm. Math. Helv. 53 (1978), 301-321. MR 81i:30041

[Su] D. Sullivan, Quasiconformal homeomorphisms and dynamics I: Solution of the Fatou-Julia problem on wandering domains, Ann. of Math. 122 (1985), 401-418. MR 87i:58103

Institute of Mathematics, Academy of Mathematics and System Sciences, Chinese Academy of Sciences, Beijing 100080, People's Republic of China

E-mail address: gzcui@math08.math.ac.cn 